\title{
Relationship Between Baseline Influenza-like IIIness Rates And Healthcare Settings
}

\author{
Dino P. Rumoro', Shital C. Shah¹, Gillian S. Gibbs ${ }^{1}$, Marilyn M. Hallock, \\ Gordon M. Trenholme ${ }^{1}$ and Michael J. Waddell ${ }^{2}$
}

${ }^{1}$ Emergency Medicine, Rush University Medical Center, Chicago, IL, USA; ²Pangaea Information Technologies, Chicago, IL, USA

\section{Objective}

To examine the baseline influenza-like illness (ILI) rates in the emergency departments (ED) of a large academic medical center (AMC), community hospital $(\mathrm{CH})$, and neighboring adult and pediatric primary care clinics.

\section{Introduction}

The primary goal of syndromic surveillance is early recognition of disease trends, in order to identify and control infectious disease outbreaks, such as influenza. For surveillance of influenza-like illness (ILI), public health departments receive data from multiple sources with varying degrees of patient acuity, including outpatient clinics and emergency departments. However, the lack of standardization of these data sources may lead to varying baseline levels of ILI activity within a local area.

\section{Methods}

Geographic Utilization of Artificial Intelligence in Real-Time for Disease Identification and Alert Notification (GUARDIAN) - a syndromic surveillance program - was used to automate ILI detection using free text chief complaint/reason for visit fields and vital signs for a large AMC - ED, CH - ED, and neighboring outpatient clinics during the summer (June 15, 2016 to August 18, 2016) in order to create a baseline. The GUARDIAN system defined ILI as fever (temperature $\geq 100^{\circ} \mathrm{F}$ ) and cough and/or sore throat. Descriptive analysis of the observed ILI rates along with bivariate ANOVA with post hoc Bonferroni and t-test were utilized to examine the difference within the settings.

\section{Results}

The average ILI rate for EDs is higher than the clinics by at least $0.39 \%$. The $\mathrm{CH}-\mathrm{ED}$ had $4.23 \%$ baseline ILI rate as compared to $1.35 \%$ for AMC-ED. While the AMC - Clinics have $0.96 \%$ baseline ILI rate as compared to $0.25 \%$ for $\mathrm{CH}-\mathrm{Clinics}$. The $\mathrm{CH}-\mathrm{ED}$ and $\mathrm{AMC}-\mathrm{Clinics}$ represented higher variations. Based on bivariate test, $\mathrm{CH}-\mathrm{ED}$ was significantly different than AMC - ED, AMC - Clinics, and $\mathrm{CH}-$ Clinics $(\mathrm{F}=10.58, \mathrm{df}=1238, \mathrm{p}<0.05)$. For the AMC Clinics, the average ILI rate for clinics providing services to adult patients was $0.66 \%$ (SD: $4.5 \%$ ) as compared to $2.03 \%$ (SD: $10.81 \%$ ) for pediatric clinics, which was not statistically significant.

\section{Conclusions}

The CH - ED has higher baseline ILI rates compared to other settings, as well as the CDC Region 5's baseline (1.9\% for 20152016). Based on previous studies ${ }^{1}$, this is likely due to providers' use of chief complaint free text fields. Thus, the $\mathrm{CH}-\mathrm{ED}$ will have higher thresholds for widespread ILI activity. In addition, differences in baseline ILI rates between AMC - ED, AMC - Clinics, and CH Clinics may result in different thresholds for widespread ILI activity (i.e., Average +3 Standard Deviations). The $\mathrm{CH}-\mathrm{ED}$ and AMC Clinics had higher baseline standard deviations, indicting variations in underlying patient populations. In addition, pediatric clinics have higher baseline ILI activity but also higher variations, indicating the unique characteristics of pediatric patients. Thus, due to the above findings, there is a need to closely monitor the ILI rates at various healthcare sites for both timing of onset, as well as the intensity of ILI activity.

Table 1. Baseline influenza-like illness (ILI) rates during summer 2016

\begin{tabular}{|c|c|c|c|}
\hline Healthcare Setting & Total Encounters & Average ILI Rate & Standard Deviation \\
\hline AMC - ED & 12,840 & $1.35 \%$ & $1.11 \%$ \\
\hline CH - ED & 6,428 & $4.23 \%$ & $4.10 \%$ \\
\hline AMC - Clinics & 25,935 & $0.96 \%$ & $6.45 \%$ \\
\hline CH - Clinics & 8,998 & $0.25 \%$ & $0.90 \%$ \\
\hline Overall & 54,201 & $0.97 \%$ & $5.31 \%$ \\
\hline
\end{tabular}

Note: $\mathrm{AMC}=$ Academic Medical Center and $\mathrm{CH}=$ Community Hospital ANOVA with post hoc Bonferroni results $\mathrm{CH}$ - ED significantly different than AMC - ED, AMC Clinics, and CH - Clinics

\section{Keywords}

GUARDIAN; Influenza-like illness; Baseline ILI rates

\section{Acknowledgments}

GUARDIAN is funded by the US Department of Defense, Telemedicine and Advanced Technology Research Center, Award numbers W81XWH-09-1-0662 and W81XWH-11-1-0711.

\section{References}

1. Rumoro, D., Shah, S., Hallock, M., Gibbs, G., Trenholme, G., Waddell, M., \& Bernstein, J. The impact of documentation style on influenzalike illness rates in the emergency department [Abstract]. Online Journal of Public Health Informatics. 2016;8(1) e35.

*Shital C. Shah

E-mail:Shital_Shah@rush.edu 